\title{
Activation of the human hippocampal formation during auditory-verbal long-term memory function
}

\author{
P.M. Grasby ${ }^{\mathrm{a}, \mathrm{b}, *}$, C.D. Frith ${ }^{\mathrm{a} . \mathrm{c}}$, K. Friston ${ }^{\mathrm{a}}$, R.S.J. Frackowiak ${ }^{\mathrm{a}, \mathrm{d}}$, R.J. Dolan ${ }^{\mathrm{a}, \mathrm{b}, \mathrm{d}}$ \\ ${ }^{\prime} M R C$ Cyclotron Unit, Hammersmith Hospital, London W12 OHS, UK \\ ${ }^{h}$ Royal Free Hospital School of Medicine, London NW3 2QG, UK \\ 'Department of Psychology, University. College, London, UK \\ "National Hospital for Neurology and Neurosurgery. London WCIN $3 B G, U K$
}

(Received 23 June 1993; Revised version received 28 July 1993; Accepted 23 August 1993)

Key words: Hippocampal formation; Memory; Positron emission tomography

\begin{abstract}
Clinical data from brain-damaged patients implicates the human hippocampal formation in memory function. We tested the hypothesis that long-term memory function is associated with activation of the hippocampal formation in humans by measuring regional cerebral blood flow changes whilst subjects performed memory tasks. Bilateral hippocampal regional cerebral blood flow was significantly correlated with a measure of the engagement of long-term auditory -verbal memory. No such association was observed for the degree of engagement of auditory-verbal subspan memory. These data provide, for the first time, direct in vivo evidence for the involvement of the hippocampal formation in long-term memory in the intact human brain.
\end{abstract}

Neuropsychological studies of amnesic patients with localized brain damage have been the predominant method for establishing the neural correlates of normal memory function. However, the inherent difficulty of this "lesion model" lies in relating memory function to the integrated functional anatomy of the intact living brain. Using this anatomical-clinical correlation approach, the human hippocampus has a long, though contentious, role in memory function $[9,13]$. Bilateral damage to the medial aspect of the temporal lobe, including the hippocampus, produces characteristic impairments in memory function when task demands exceed the immediate span of short-term memory $[1,11,13]$. Such "supraspan" memory tasks include, for example, the free recall of heard word lists, typically $10-15$ words presented over 20-30 s. If a normal subject's recall is plotted against the position of the word in the list (serial position curve), words are preferentially recalled from the beginning and end of the list; so-called "primacy" and "recency effects" [14]. The recency effect is dependent on a short-term memory system of limited capacity [18]. Performance on the mid-portion of the serial position curve reflects the operation of long-term memory function [2] whilst the primacy effect may relate to the effects of re-

\footnotetext{
*Corresponding author
}

hearsal as well as long-term memory function [2]. In patients with medial temporal lobe damage, the recall of items from the beginning and mid-portion of such word lists is impaired whereas items from the end of the list are recalled normally [1]. Such neuropsychological data indicate a role for the human hippocampus in processing information that exceeds the span of short-term memory. This span has been shown to be approximately five words or seven digits [10].

The ability to relate activity in the hippocampal formation to specific aspects of psychological function will be of considerable importance in clarifying its precise role in mnemonic processing. Functional brain-imaging techniques using positron-emission tomography (PET) now provide an alternative to the "lesion model" in localizing the anatomical correlates of higher mental functions [8]. With these techniques neuronal activation can be indexed by task-related changes in regional cerebral blood flow $(\mathrm{rCBF})$ in the intact brain. A task-related change in $\mathrm{rCBF}$ can be defined by the difference in $\mathrm{rCBF}$ profiles between two brain scans when a single cognitive component is altered (cognitive subtraction) or by the correlation of $\mathrm{rCBF}$ with the systematic variation of a single task parameter across a number of brain scans (graded response). We describe an experiment using a graded response auditory-verbal memory task that di- 
rectly addressed the relationship between memory function and hippocampal activity in vivo. We based our experimental design on the hypothesis that a performancederived measure of long-term memory function would correlate with hippocampal rCBF whilst a performancederived measure of subspan memory would not.

Six male right-handed volunteers (aged from 21 to 36 yr) gave written informed consent. Each subject underwent 12 serial $\mathrm{rCBF}$ measurements over a 3 -h period with a CTI 953B-PET scanner (CTI, Knoxville, TN) with collimating septa retracted. For each $\mathrm{rCBF}$ measurement, $\mathrm{H}_{2}{ }^{15} \mathrm{O}$ was given as an infusion over $120 \mathrm{~s}$. The amount of tracer injected per subject was $135 \mathrm{mCi}$ (calculated dosimetry $5 \mathrm{mSv}$ ). The integrated radioactivity counts accumulated over a 165 -s period during the performance of the auditory-verbal memory task was used as an index of rCBF [7]. Subjects were asked to remember and immediately and freely recall a series of heard word lists (one word every $2 \mathrm{~s}$ ) that varied from two to 13 words in length. Word-list length was randomized amongst scans and a total of 60-66 different words were presented during each scan. Within a single scan, wordlist length was kept constant. For example, in the first scan for a single subject, eight different eight-word lists were presented, for the fifth scan 20 different three-word lists were presented.

PET images were reconstructed using a validated algorithm for full 3-D reconstruction with retractable septa [17]. Images were displayed in ANALYZE (BRU; Mayo Foundation, Rochester, MN). Statistical analysis was performed in PROMATLAB (MathWorks, Natick, MA) using statistical parametric mapping [4] (SPM; MRC Cyclotron Unit, London, UK). Following realignment [19] and stereotactic normalization $[3,6]$ into the atlas of Tailairach and Tournoux [16], all scans were subject to an ANCOVA [5]. For each pixel, the ANCOVA generated 12 condition-specific mean rCBF values, one for each word-list length, each with an associated error variance. With appropriate weighted contrasts reflecting memory performance, areas with significant correlations between $\mathrm{rCBF}$ and memory performance were identified. Data from the middle portion of the serial position curve (words correctly recalled without contamination by primacy or recency components) were used to generate a measure of memory performance that represented a pure engagement of long-term memory processes [2] (Table I). Only data from the "supraspan" scans of seven or more words in length were used. In these word lists, the primacy effect was conservatively assumed to involve the first two words presented and the recency effect the last four words in any word list. This left a variable midportion of the serial position from one word in the sevenword list to seven words in the 13-word list from which scores for long-term memory performance (total number of words recalled) were calculated. Separate correlations of $\mathrm{rCBF}$ with the total number of words correctly recalled in the "short word lists" were also made as an index of subspan memory engagement. These scans were of word-list length two, three, four, five, six and seven words (for the seven-word list the third word in the list was ignored as it was used as a measure of long-term memory). For all weighted contrasts, a threshold of $P<0.01$ was used (not corrected for multiple comparisons). This statistical level was justified as our hypothesis was constrained to a specific area in the whole scanned volume, i.e., the hippocampal formation.

The upper images of Fig. 1 show statistical parametric maps of transverse sections through the hippocampal and parahippocampal regions. Brain areas showing significant correlations of rCBF with subspan memory performance (upper) and long-term memory performance (middle) are shown. As predicted the number of words correctly recalled from the mid-portion of the serial position curve correlated with increased blood flow bilaterally in the hippocampal formation. The spatial extent of this correlation appeared greater on the left than right (see Fig. 1). The site of maximal correlation was located in the left hippocampus (co-ordinates $-24,-30,-4$ in the $x, y$ and $z$ planes of the Talairach and Tournoux atlas) (correlation coefficient for left hippocampus $=0.68$ $P<0.05$ Pearson product moment correlation) (see Fig. 2). In addition, significant correlations were seen in the region of the posterior hypothalamus, posterior cingulate, the precuneus and angular gyrus (data not shown).

\section{TABLE I}

\section{MEMORY PERFORMANCE}

Data used in the weighted contrasts of correlations between $\mathrm{rCBF}$ and memory performance. (a) wordlist length presented to subjects; (b) total number of words correctly recalled; (c) long-term memory engagement $=$ number of words correctly recalled from the mid-portion of the serial position curve; (d) number of words correctly recalled from the subspan conditions.

\begin{tabular}{rlll}
$a$ & $b$ & $c$ & $d$ \\
\hline 2 & 2.0 & - & 2.0 \\
3 & 3.0 & - & 3.0 \\
4 & 4.0 & - & 4.0 \\
5 & 4.8 & - & 4.8 \\
6 & 5.6 & - & 5.6 \\
7 & 6.2 & 0.9 & 5.5 \\
8 & 6.8 & 1.7 & - \\
9 & 6.9 & 2.2 & - \\
10 & 7.1 & 2.5 & - \\
11 & 7.7 & 3.0 & - \\
12 & 7.1 & 3.2 & - \\
13 & 8.0 & 4.3 & - \\
\hline
\end{tabular}




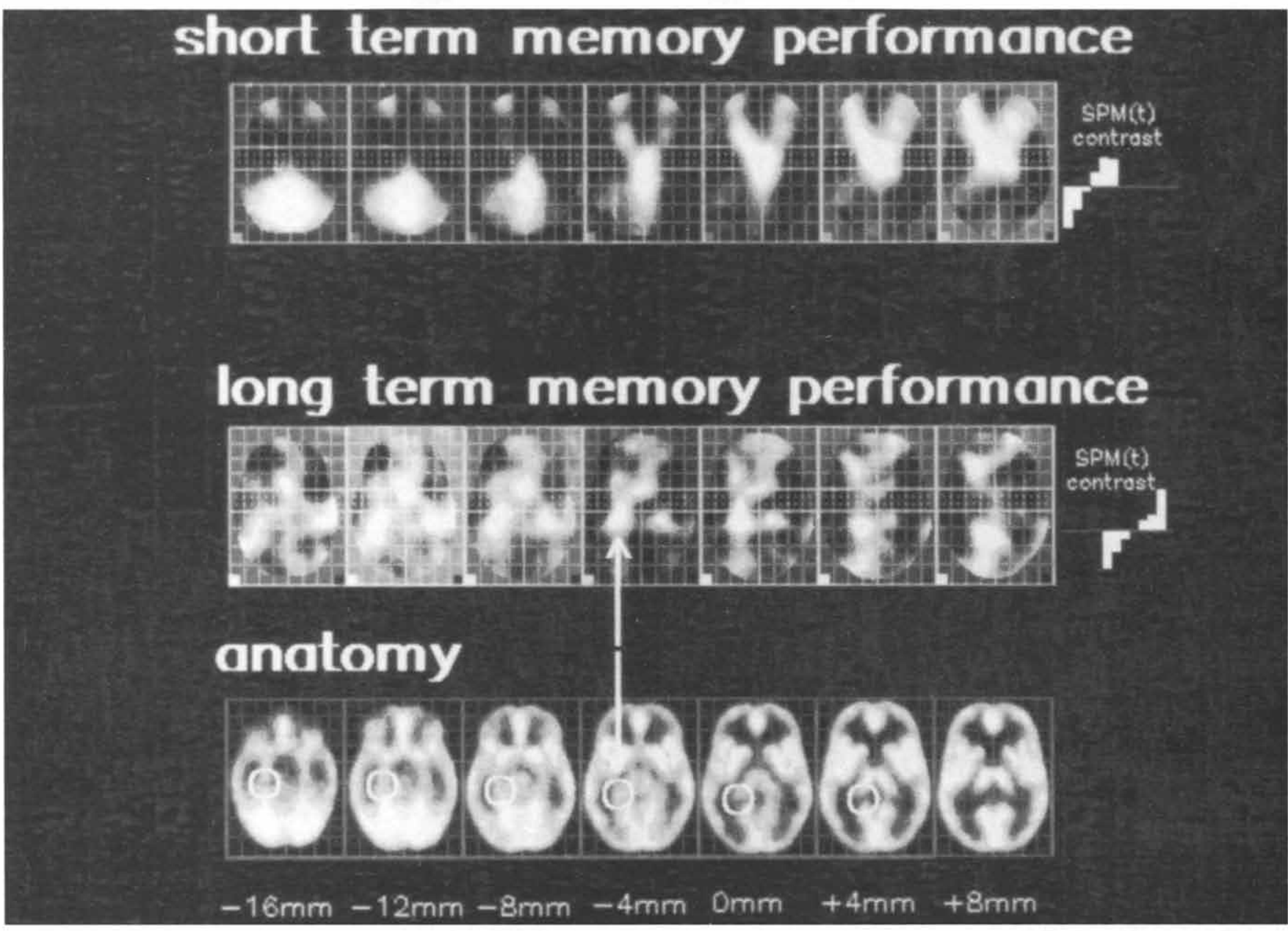

Fig. 1. Statistical parametric maps of transverse sections through the hippocampal and parahippocampal regions from plane $z=-16 \mathrm{~mm}$ to $+8 \mathrm{~mm}$ relative to the anterior-posterior commissure line. Brain areas showing significant correlations of rCBF with subspan memory performance (upper) and long-term memory performance (middle) are shown. The coloured square at the lower left corner on each plane represents an omnibus threshold of significance of $P<0.01$. The lower images are averaged PET images from a database of 20 subjects through the same planes to illustrate the relevant anatomical regions. The hippocampal and parahippocampal region is circled for illustrative purposes on these images. The arrows at $-4 \mathrm{~mm}$ illustrate the correspondence between the hippocampal/parahippocampal region and the focus of maximal correlation. The linear contrasts to the right of each row of transverse images show the relative weighting applied to each scan for the comparisons made. Weightings were applied directly from the measures of long-term and subspan memory performance in Table I.

In contrast to our measure of long- term memory performance, the number of words correctly recalled in the subspan word lists did not correlate with hippocampal rCBF at the same location (correlation coefficient for left hippocampus $=0.173, P>0.05$ Pearson product moment correlation). This suggests that the observed relationship is specific for long-term memory processes rather than a non-specific aspect of memory performance that is common to both tasks. In addition, intrusion errors from previous word lists were very rare thus interference effects are unlikely to be a confounding factor in this study.

The lack of correlation between hippocampal rCBF and performance in the subspan tasks confirms clinical and recent PET studies showing that such memory tasks are independent of medial temporal lobe function [12, 15]. Hippocampal engagement was detected when task demand exceeded the capacity of short-term memory processes. Clinical and experimental data suggests hippocampal function is essential for the encoding and consolidation of long-term memory traces at a neocortical level whilst the retrieval of such memory traces can occur independently of hippocampal function [15]. In the light of this view, the correlation of hippocampal rCBF with long-term memory function probably reflects neural activity associated with encoding and consolidation. Further experiments exploring the encoding, consolidation and retrieval phases of word-list learning separately will address this hypothesis. The present results demonstrate convergent and complementary evidence to that derived 


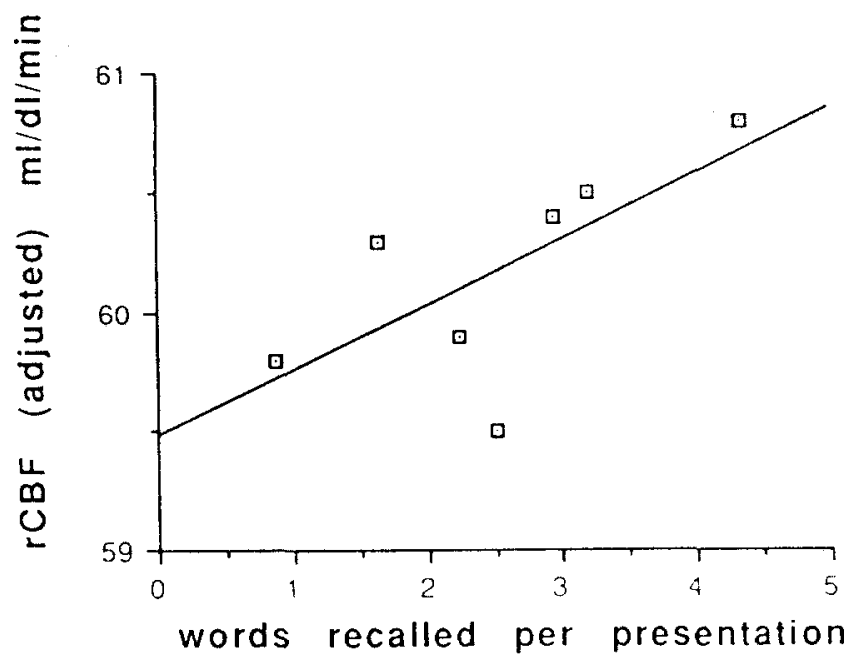

Fig. 2. (a) Mean $\mathrm{rCBF}$ data from the left hippocampal region for all subjects vs. mean number of words per presentation correctly recalled from the mid-portion of the serial position curve (see text for details). The precise co-ordinates are $-24,-30,-4 \mathrm{~mm}$ in the $x, y$ and $z$ planes of the Talairach and Tournoux atlas.

from "lesion models", that a structure, the hippocampus, associated with long-term memory function, is indeed activated in the intact human brain.

1 Baddeley, A.D. and Warrington, E.K., Amnesia and the distinction between long- and short-term memory, J. Verb. Learn. Verb. Behav., 9 (1970) 176-189.

2 Capitani, E., Sala, S.D., Logie, R.H. and Spinnler, H., Recency, primacy, and memory: reappraising and standardising the serial position curve, Cortex, 28 (1992) 315-342.

3 Friston, K.J., Frith, C.D., Liddle, P.F. and Frackowiak, R.S.J., Plastic transformation of PET images, J. Comput. Assist. Tomogr., 15 (1991) 634-639.

4 Friston, K.J., Frith, C.D., Liddle, P.F. and Frackowiak, R.S.J., Comparing functional (PET) images: the assessment of significant change, J. Cereb. Blood Flow Metab., 11 (1991) 690-699.

5 Friston, K.J., Frith, C.D., Liddle, P.F., Lammertsma, A.A., Dolan, R.J. and Frackowiak, R.S.J., The relationship between local and global changes in PET scans, J. Cereb. Blood Flow Metab., 10 (1990) 458-466.

6 Friston, K.J., Passingham, R.E., Nutt, J.G., Heather, J.D., Sawle, G.V. and Frackowiak, R.S.J., Localization in PET images: direct fitting of the intercommissural (AC-PC) line, J. Cereb. Blood Flow Metab., 9 (1989) 690-695.

7 Fox, P.T. and Mintum, M.A., Non-invasive functional brain mapping by change distribution analysis of averaged PET images of $\mathrm{H}_{2}{ }^{15} \mathrm{O}$ tissue activity, J. Nucl. Med., 30 (1989) 141-149.

8 Grasby, P.M., Frith, C.D., Friston, K.J., Bench, C., Frackowiak, R.S.J. and Dolan, R.J., Functional mapping of brain areas implicated in auditory-verbal memory function, Brain, 116 (1993) 1-20.

9 Mayes, A.R., Memory and the hippocampus, Curr. Opin. Neurol. Neurosurg., 4 (1991) 115-120.

10 Miller, G.A., The magical number seven: plus or minus two. Some limits on our capacity for processing information, Psychol. Rev., 9 (1956) $81-97$

11 Milner, B., Hemispheric specialization: scope and limits. In F.O. Schmitt and F.G. Worden (Eds.), The Neurosciences: Third Study Program, MIT Press, Cambridge, MA, 1974, pp. 75-89.

12 Paulesu, E., Frith, C.D. and Frackowiak, R.S.J., The neural correlates of the verbal component of working memory, Nature, 362 (1993) 342-344.

13 Scoville, W.B. and Milner, B., Loss of recent memory after bilateral hippocampal lesions, J. Neurol. Neurosurg. Psychiatr., 20 (1957) $11-21$.

14 Squire, L.R., Memory: neural organiation and behaviour. In F. Plum and V.B. Mountcastle (Eds.), Handbook of Physiology - The Nervous System, Williams and Wilkins, Baltimore, MD, 1987, pp. 295-371.

15 Squire, L.R. and Zola-Morgan, S., The medial temporal lobe memory system, Science, 253 (1991) 1380-1386.

16 Talaraich, J. and Tournoux, P., A Co-Planar Stereotactic Atlas of a Human Brain, Thieme Verlag, Stuttgart, Germany, 1988.

17 Townsend, D.W., Geissbuhler, A., Defrise, M., Hoffman, E.J., Spinks, T.J., Bailey, D.L., Gilardi, M.C. and Jones, T., Fully threedimensional reconstruction for a PET camera with retractable septa, IEEE Trans. Med. Imag., 10 (1991) 505 512.

18 Vallar, G. and Papagno, C., Phonological short-term store and the nature of the recency effect: evidence from neuropsychology, Brain Cogn., 5 (1986) 428-442.

19 Woods, R.D., Cherry, S.R. and Mazziota, J.C., A rapid automated algorithm for accurately aligning and reslicing positron emission tomography images, J. Comput. Assist. Tomogr., 16 (1992) 620633. 